\title{
Large-amplitude capillary waves in electrified fluid sheets
}

\author{
By DEMETRIOS T. PAPAGEORGIOU ${ }^{1}$ \\ AND JEAN-M ARC VANDEN-BROECK ${ }^{2}$ \\ ${ }^{1}$ Department of Mathematical Sciences and Center for Applied Mathematics and Statistics, New Jersey \\ Institute of Technology, Newark, NJ 07102, USA \\ ${ }^{2}$ School of Mathematics, University of East Anglia, Norwich NR4 7TJ, UK
}

(Received 16 June and in revised form 18 September 2003)

Large-amplitude capillary waves on fluid sheets are computed in the presence of a uniform electric field acting in a direction parallel to the undisturbed configuration. The fluid is taken to be inviscid, incompressible and non-conducting. Travelling waves of arbitrary amplitudes and wavelengths are calculated and the effect of the electric field is studied. The solutions found generalize the exact symmetric solutions of Kinnersley (1976) to include electric fields, for which no exact solutions have been found. Long-wave nonlinear waves are also constructed using asymptotic methods. The asymptotic solutions are compared with the full computations as the wavelength increases, and agreement is found to be excellent.

\section{Introduction}

Liquid films arise in many diverse physical applications including cooling systems, coating processes and combustion, as well as biological applications. The fundamental problem of the stability and nonlinear states of such systems is of interest since the dynamics can enhance quantities such as heat or mass transfer coefficients. Heat transfer enhancement was found by Miyara (1999) who studied theoretically the heat transfer coefficient through a falling film that supports travelling waves. It was found that interfacial waves enhance heat transfer by film thinning and convection effects. Additional evidence of this can be found in other related work, for example, Shmerler \& Mudawar (1987) and the more recent experimental study of Al-Sibai, Leefken, Renz (2002) (see references therein also).

Here we consider the dynamics of two-dimensional free liquid films in the presence of an electric field. A related problem involves normal electric fields to control heat transfer in devices incorporating perfectly conducting film flow on inclined planes see Gonzalez \& Castellanos (1996). There have been numerous theoretical studies of the linear stability of electrified liquid films. The case of two conducting or nonconducting fluids of infinite extent separated by a sharp interface has been considered by Melcher \& Schwarz (1968). They consider a constant electric field applied in the plane of the undisturbed interface and study the linear stability of viscous and inviscid fluids. The presence of the electric field produces a dispersive regularization of short waves; similar findings are reported by El-Sayed (1999) who considers the linear stability problem in the presence of an air stream outside the sheet, hence allowing Kelvin-Helmholtz instability also. Melcher \& Schwarz (1968) also point out that the electric field produces a net force due to polarization, which tends to displace the interface from its flat configuration. 
In this work we consider a unbounded two-dimensional fluid sheet which initially has uniform thickness and is surrounded by an ambient passive fluid. We assume that the effects of gravity are negligible, a condition that is satisfied when the Bond number, $\rho g d^{2} / \sigma$, is small, where $\rho$ is the fluid density, $g$ the acceleration due to gravity, $2 d$ the undisturbed sheet thickness and $\sigma$ the surface tension coefficient - this condition can be expected to hold for a thin layer and/or large surface tension. An electric field acts in the plane of the sheet driven by a constant potential difference. For nonlinear interfacial deformations we must solve for the hydrodynamics inside the sheet and for the voltage potential inside and outside the sheet, the latter region extending laterally to infinity. The fluid and electric fields are coupled through the Maxwell stresses which modify the hydrodynamic stresses at the interface - Jackson (1963). This constitutes a nonlinear problem that must in general be addressed numerically. We commence our study of this free boundary problem by considering travelling wave solutions of arbitrary amplitude and wavelength. We restrict our attention to symmetric waves. A study of antisymmetric waves will be presented in Papageorgiou \& Vanden-Broeck (2004).

The formation of waves on liquid layers when surface tension acts has been the subject of numerous investigations. Crapper (1957) studied two-dimensional capillary waves in deep water and found a class of exact travelling wave solutions for which the interface can be a single or multi-valued function. Crapper's analysis was extended by Kinnersley (1976) to the case of fluid layers and exact capillary waves are found for both symmetric and asymmetric elevations (since the problem is inviscid, the symmetric solutions also represent the flow over a solid substrate). Further exact solutions were recently found by Crowdy (1999). When gravity is also present exact solutions are not possible. In the absence of surface tension, there exists a vast literature devoted to the study of nonlinear gravity waves starting from the work of Stokes (1847) and the more recent studies of Schwartz (1974) and Longuet-Higgins (1975) among others. Gravity-capillary waves in irrotational fluids have been computed numerically by a number of authors including Schwartz \& Vanden-Broeck (1979), Chen \& Saffman (1979), Hogan (1980) and Hunter \& Vanden-Broeck (1983), while calculations in the presence of vorticity are reported in Kang \& Vanden-Broeck (2000).

Long-wave models have been used to develop nonlinear evolution equations for the sheet (see for example Erneux \& Davis 1993 for viscous flows with Van der Waals forces; Ida \& Miksis $1998 a, b$ for derivation in non-flat geometries; Ida \& Miksis 1996 and Vaynblat, Lister \& Witelski 2001 for computations of the two-dimensional evolution equations). Of particular interest to the present study is the inviscid problem. The long-wave evolution equations can be deduced directly from those of Erneux \& Davis (1993) by dropping viscous and van der Waals forces and keeping surface tension. This system was derived independently by Matsuuchi $(1974,1976)$ at about the same time that the exact solutions of Kinnersley (1976) were given. Matsuuchi also constructed nonlinear travelling wave solutions which are the long-wave analogues of the exact Kinnersley waves. Stability of the travelling waves is considered by Matsuuchi (1976), and modulational instability is predicted and confirmed numerically. The long-wave inviscid problem has also been studied more recently and more extensively by Mehring \& Sirignano (1999) who also allow sinuous modes in their evolution equations. Numerical solutions of the long-wave system are compared with solutions of the Euler equations by the vortex blob method, and good agreement is found. These authors also report calculations that lead to rupture but the pinching solutions are not studied in detail. It is interesting that the inviscid long-wave model with surface tension is also suggested by Pugh \& Shelley (1998) in the context of 
vortex sheet roll-up. These authors study the singularity formation in detail and construct similarity solutions guided by the calculations.

The inclusion of an electric field into the long-wave models for inviscid and viscous sheets (including Van der Waals forces) was recently accomplished by Tilley, Petropoulos \& Papageorgiou (2001) and Savettaseranee et al. (2003), respectively. The electric field exerts Maxwell stresses at the interface due to the difference in dielectric properties inside and outside the sheet. Using matched asymptotic expansions, Tilley et al. (2001) show that the electric field modifies the evolution system described above, by adding a non-local term to the Bernoulli equation proportional to the Hilbert transform of the leading-order sheet curvature. The authors study this system numerically and construct travelling wave solutions and study their stability. They also study the initial value problem in order to evaluate the effects of the electric field on the rupture solutions of Pugh \& Shelley (1998). It is found that the electric field delays the time of rupture but does not affect the singularity structure, to leading order. In the case of viscous films with Van der Waals forces, it is predicted analytically and confirmed numerically that a large enough electric field will prevent rupture events which would otherwise be present (see Savettaseranee et al. 2003 for details).

The full problem when an electric field acts has not been studied. In the present work we make no approximations for the competing length scales and address the problem of capillary waves driven by an electric field parallel to the fluid layer. The internal and external electric fields are fully accounted for, as is the incompressible irrotational fluid motion inside the sheet. We use boundary integral methods to construct travelling waves of arbitrary amplitudes and periods and study the effects of the physical parameters on these waves. At large wavelengths, an asymptotic theory is developed starting from the boundary integral formulation and the results of Tilley et al. (2001) are recovered. The asymptotic and exact solutions are compared and agreement is found to be excellent even for waves which are not too long. We can conclude, therefore, that the stability results of the nonlinear travelling waves constructed by Tilley et al. (2001) extend to the fully nonlinear ones computed here. Note that the flat state is linearly temporally stable for this problem and our interest is in the construction of fully nonlinear travelling waves.

The article is organized as follows. Section 2 describes the governing equations and gives the exact dimensionless problem. Section 3 reformulates the problem in terms of integral equations and $\S 4$ contains the asymptotic analysis in the limit of long waves. Section 5 presents numerical solutions for a range of physical parameters and comparisons between asymptotic and computed solutions. Section 6 is devoted to concluding remarks.

\section{Governing equations}

Consider an inviscid, incompressible and irrotational flow in a liquid layer of undisturbed thickness $2 d$ which is bounded symmetrically by free surfaces at $y= \pm S$, say (see figure 1). Surface tension with coefficient $\sigma$ acts at the interfaces and gravity is ignored (the latter assumption holds if the Bond number, $\left.\left(\rho g d^{2}\right) / \sigma \ll 1\right)$. An applied uniform electric field given by $\boldsymbol{E}_{0}=\left(V_{0} / 2 L\right) \boldsymbol{i}$, also acts, where $V_{0}$ is the characteristic voltage drop over a characteristic distance $L$. We introduce dimensional fluid and voltage potentials $\phi$ and $V^{(1,2)}$ respectively; the field equations are Laplace's equation

$$
\begin{aligned}
\nabla^{2} \phi(x, y ; t) & =0, \\
\nabla^{2} V^{(1,2)}(x, y ; t) & =0,
\end{aligned}
$$




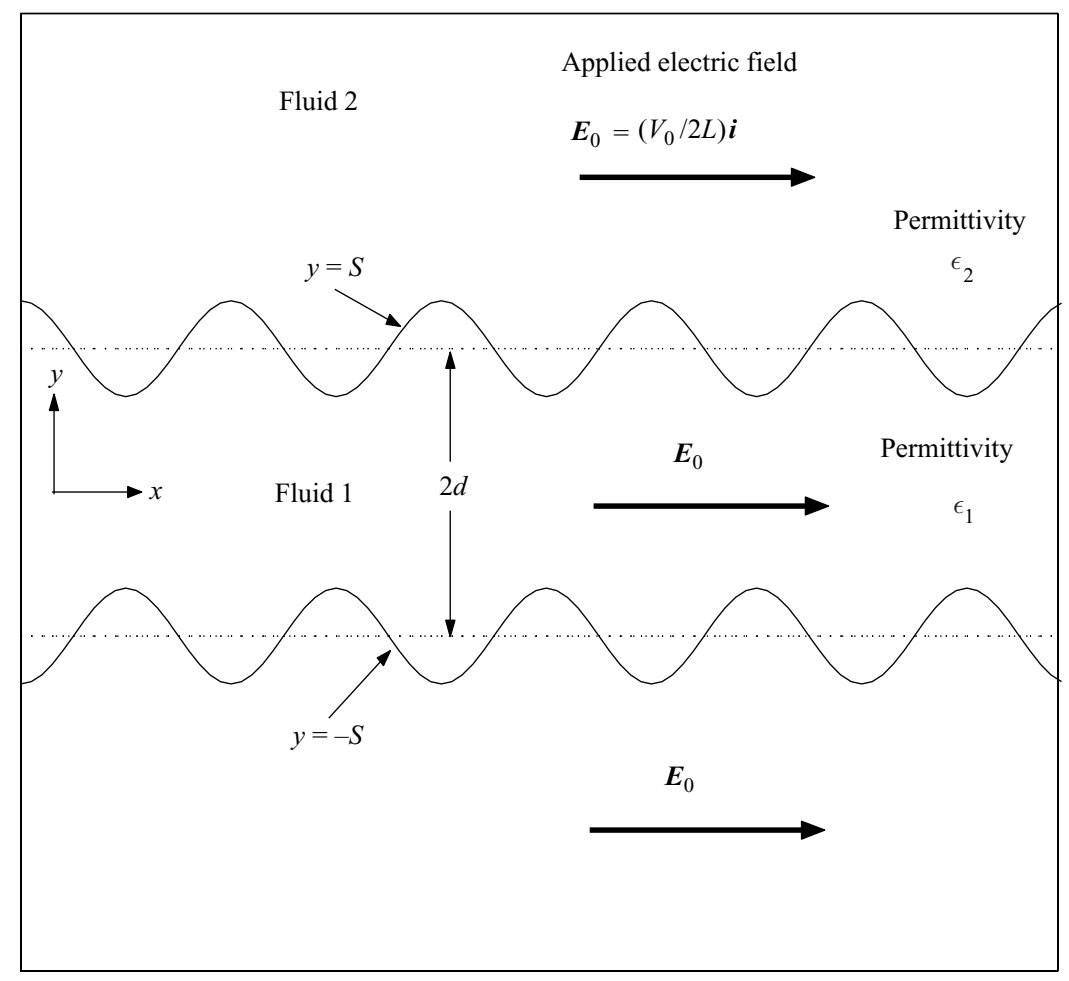

FIGURE 1. Schematic of the problem. The undisturbed layer has thickness $2 d$ and region 2 extends to infinity.

where in what follows superscripts 1,2 respectively denote the regions bounded by and outside of the moving interfaces (see figure 1). The electric fields are $\boldsymbol{E}^{(1,2)}=\nabla V^{(1,2)}$.

Symmetric solutions about the centreline $y=0$, are considered, which yield the boundary conditions $\phi_{y}=V_{y}^{(1)}=0$. As $|y| \rightarrow \infty, \partial V^{(2)} / \partial x \rightarrow V_{0} /(2 L)$. We note that for inviscid flows the symmetry condition describes symmetric liquid layer flows or flow over a flat solid surface. When electric fields parallel to the undisturbed sheet are imposed, however, the presence of a solid substrate changes the undisturbed field lines and results in a different problem. Here we study the liquid layer problem.

The boundary conditions at the free surface $y=S$ (only half the domain is considered) are the kinematic condition, continuity of normal stresses, and the continuity of the normal component of the displacement field $(\epsilon \boldsymbol{E})$ and tangential components of the electric field. These are, at $y=S(x, t)$,

$$
\begin{aligned}
S_{t}+\phi_{x} S_{x}-\phi_{y} & =0 \\
{[\boldsymbol{n} \cdot \boldsymbol{T} \cdot \boldsymbol{n}]_{2}^{1} } & =\sigma \operatorname{div} \boldsymbol{n}, \\
{[\epsilon \boldsymbol{E} \cdot \boldsymbol{n}]_{2}^{1} } & =0, \\
{[\boldsymbol{E} \cdot \boldsymbol{t}]_{2}^{1} } & =0,
\end{aligned}
$$

where $[\cdot]_{2}^{1}$ denotes the jump in the quantity as the interface is crossed from the fluid region, the vectors $\boldsymbol{n}, \boldsymbol{t}$ are the outward-pointing normal and tangent to the interface respectively, and the stress tensor $\boldsymbol{T}$ is given by

$$
\begin{aligned}
T_{i j} & =-p \delta_{i j}+\mathscr{E}_{i j}, \\
\mathscr{E}_{i j} & =\epsilon\left(E_{i} E_{j}-\frac{1}{2}|\boldsymbol{E}|^{2} \delta_{i j}\right) .
\end{aligned}
$$


The first term in (2.7) is the inviscid hydrodynamic contribution and the second term arises from interfacial electric field stresses given by the Maxwell stress tensor (see Jackson 1963, Chap. 6). The parameters $\epsilon_{1}, \epsilon_{2}$ are the dielectric constants in regions 1 and 2 respectively.

The momentum equations can be integrated to yield a Bernoulli equation at the interface. The pressure in region 2 is ambient and equal to a constant, and on elimination of the pressure jump across the interface from (2.4), we arrive at the following Bernoulli boundary condition:

$$
\rho_{1}\left(\phi_{t}+\frac{1}{2}|\nabla \phi|^{2}\right)+\frac{1}{1+S_{x}^{2}}\left\{S_{x}^{2}\left[\mathscr{E}_{11}\right]_{2}^{1}-2 S_{x}\left[\mathscr{E}_{12}\right]_{2}^{1}+\left[\mathscr{E}_{22}\right]_{2}^{1}\right\}=\frac{\sigma S_{x x}}{\left(1+S_{x}^{2}\right)^{3 / 2}}+K_{p}
$$

The Maxwell stresses appearing in (2.8) are given by

$$
\mathscr{E}_{11}=\frac{\epsilon}{2}\left(V_{x}^{2}-V_{y}^{2}\right), \quad \mathscr{E}_{12}=\epsilon V_{x} V_{y}, \quad \mathscr{E}_{22}=\frac{\epsilon}{2}\left(V_{y}^{2}-V_{x}^{2}\right),
$$

and superscripts 1 and 2 are implied where appropriate in (2.9). For the undisturbed state $(\phi=0, S=d)$, the constant $K_{p}=\frac{1}{8} V_{0}^{2}\left(\epsilon_{2}-\epsilon_{1}\right)$. This constant is related to the polarization pressure induced on fluid interfaces by the electric field (see Melcher \& Schwarz 1968), and can be eliminated by differentiation of (2.8) with respect to $x$, for example.

\subsection{Dimensionless equations}

The equations and boundary conditions are made dimensionless using an outer length scale $L$ to measure distances:

$x=L x^{\prime}, \quad y=L y^{\prime}, \quad S=L S^{\prime}, \quad t=\sqrt{\frac{\rho_{1} L^{3}}{\sigma}} t^{\prime}, \quad \phi=\sqrt{\frac{\sigma L}{\rho_{1}}} \phi^{\prime}, \quad V=V_{0} V^{\prime}$.

We substitute (2.10) into the governing equations (2.1)-(2.2) and interfacial conditions (2.3)-(2.6), and drop the primes. With this choice of reference scales the undisturbed interface is located at $y=d / L=\bar{d}$. The fluid and voltage potentials satisfy the Laplace equations

$$
\begin{aligned}
\phi_{x x}+\phi_{y y} & =0, \\
V_{x x}^{(1,2)}+V_{y y}^{(1,2)} & =0 .
\end{aligned}
$$

The kinematic, Bernoulli and continuity of the normal displacement field and tangential electric field boundary conditions, evaluated at $y=S(x, t)$, become

$$
\begin{gathered}
S_{t}+\phi_{x} S_{x}-\phi_{y}=0 \\
\phi_{t}+\frac{1}{2}\left(\phi_{x}^{2}+\phi_{y}^{2}\right)+\frac{E_{b}}{1+S_{x}^{2}}\left\{\frac{1}{2} S_{x}^{2}\left[\mathscr{M}_{11}\right]_{2}^{1}-2 S_{x}\left[\mathscr{M}_{12}\right]_{2}^{1}+\frac{1}{2}\left[\mathscr{M}_{22}\right]_{2}^{1}\right\}=\frac{S_{x x}}{\left(1+S_{x}^{2}\right)^{3 / 2}}+\bar{K}_{p} \\
\bar{\epsilon}\left(V_{y}^{(1)}-S_{x} V_{x}^{(1)}\right)=V_{y}^{(2)}-S_{x} V_{x}^{(2)} \\
V_{x}^{(1)}+S_{x} V_{y}^{(1)}=V_{x}^{(2)}+S_{x} V_{y}^{(2)}
\end{gathered}
$$

where $\bar{\epsilon}=\epsilon_{1} / \epsilon_{2}$ is the ratio of permittivities and the parameter $E_{b}=\left(\epsilon_{2} V_{0}^{2}\right) /(\sigma L)$ is the ratio of the electrically induced pressure to capillary forces; we call this an electric capillary number. For the undisturbed state, $\bar{K}_{p}=\frac{1}{8} E_{b}(1-\bar{\epsilon})$. The stress components 
in (2.14) are given by

$$
\begin{aligned}
& \mathscr{M}_{11}^{(1,2)}=\left\{\frac{\epsilon}{\epsilon_{2}}\left[V_{x}^{2}-V_{y}^{2}\right]\right\}^{(1,2)}, \\
& \mathscr{M}_{12}^{(1,2)}=\left\{\frac{\epsilon}{\epsilon_{2}} V_{x} V_{y}\right\}^{(1,2)}, \\
& \mathscr{M}_{22}^{(1,2)}=\left\{\frac{\epsilon}{\epsilon_{2}}\left[-V_{x}^{2}+V_{y}^{2}\right]\right\}^{(1,2)} .
\end{aligned}
$$

\subsection{Linear theory}

An exact solution of the dimensionless problem is $S=\bar{d}, \boldsymbol{u}=0$ and $V=x / 2$. Linearization of the system (2.11)-(2.16) about this state (the Bernoulli equation is differentiated with respect to $x$ first), and application of the symmetry conditions at $y=0$ gives the following elementary solutions for the perturbation fluid and voltage potentials:

$$
\begin{aligned}
\phi(x, y, t ; k) & =C \cosh (k y) \sin (k x-\omega t), \\
V^{(1)}(x, y, t) & =A \cosh (k y) \sin (k x-\omega t), \\
V^{(2)}(x, y, t) & =B \exp (-|k y|) \sin (k x-\omega t), \\
S(x, t) & =D \cos (k x-\omega t) .
\end{aligned}
$$

Using these solutions in the linearized kinematic, Bernoulli and electric field boundary conditions at the undisturbed level $S=\bar{d}$, gives four homogeneous equations for the constants $A, B, C, D$. This leads to the eigenrelation

$$
\omega^{2}=\frac{E_{b}(1-\bar{\epsilon})^{2}}{4} \frac{k^{3} \tanh k \bar{d}}{\bar{\epsilon} k \tanh k \bar{d}+|k|}+k^{3} \tanh k \bar{d} .
$$

In the absence of an electric field, $E_{b}=0$, we recover the linear dispersion relation for capillary waves on a fluid layer. Note that (2.24) can be used to anticipate the appropriate dispersion relation of the long-wave theory. As $\bar{d} \rightarrow 0$ (2.24) gives

$$
\omega^{2}=\frac{E_{b}(1-\bar{\epsilon})^{2}}{4} \bar{d} k^{2}|k|+\bar{d} k^{4},
$$

which is equivalent to the dispersion relation (4.29) (using $\omega^{2}=k^{2} c^{2}$ ) of the canonical long-wave evolution equations derived in $\S 4$ using formal asymptotic methods.

\section{Reformulation as integral equations}

We consider a train of waves of wavelength $\lambda$ travelling at a constant velocity $c$ in a two-fluid system. Precise definitions of $c$ and the thickness will be given later. We choose a frame of reference in which the flow is steady and we introduce Cartesian coordinates with the $x$-axis parallel to the line of symmetry and the origin at the centreline. The line of symmetry is then at $y=0$. We describe the free surface parametrically by $x=X(s)$ and $y=Y(s)$ where $s$ is the arclength. Therefore

$$
X^{\prime 2}(s)+Y^{\prime 2}(s)=1 .
$$

We choose $x=s=0$ at a crest and we express $X(s)$ and $Y(s)$ in terms of $X^{\prime}(s)$ and $Y^{\prime}(s)$ as

$$
X(s)=\int_{0}^{s} X^{\prime}(r) \mathrm{d} r
$$




$$
Y(s)=\alpha+\int_{0}^{s} Y^{\prime}(r) \mathrm{d} r .
$$

Here $\alpha$ is the ordinate of the crests.

Next we rewrite (2.14) in the new frame of reference as

$$
\begin{aligned}
\frac{1}{2}\left(\phi_{x}^{2}+\phi_{y}^{2}\right)+\frac{E_{b}}{1+S_{x}^{2}}\left\{\frac{1}{2} S_{x}^{2}\left[\mathscr{M}_{11}\right]_{2}^{1}-2 S_{x}\left[\mathscr{M}_{12}\right]_{2}^{1}+\frac{1}{2}\left[\mathscr{M}_{22}\right]_{2}^{1}\right\} \\
=Y^{\prime \prime}(s) X^{\prime}(s)-X^{\prime \prime}(s) Y^{\prime}(s)+B
\end{aligned}
$$

where $B$ is a constant to be found as part of the solution. by

The horizontal and vertical components, $u$ and $v$, of the velocity in fluid 1 are given

$$
\begin{aligned}
& u=\frac{\partial \phi}{\partial x}, \\
& v=\frac{\partial \phi}{\partial y} .
\end{aligned}
$$

We define the depth as the ordinate of the mean level of the interface. Therefore, we impose

$$
\int_{0}^{b} Y(s) X^{\prime}(s) \mathrm{d} s=\bar{d} .
$$

Here $b$ is the length of a wavelength of the interface.

We also define the velocity $c$ as the average horizontal velocity at a constant level of $y$ within the fluid. Thus we write

$$
c=\frac{1}{\lambda} \int_{0}^{\lambda} u \mathrm{~d} x
$$

where $y$ is constant. The irrotationality of the flow in region 1 implies that the value of $c$ is independent of the constant level of $y$ chosen provided it is in fluid 1 . The irrotationality of the flow implies also that (3.8) can be rewritten as

$$
c=\frac{1}{\lambda} \int_{0}^{b}\left(u X^{\prime}(s)+v Y^{\prime}(s)\right) \mathrm{d} s .
$$

As $y \rightarrow \infty, V_{x}^{(2)}$ approaches $1 / 2$. Since $V^{(2)}$ satisfies Laplace's equation, we have

$$
\int_{0}^{b}\left(V_{x}^{(2)} X^{\prime}(s)+V_{y}^{(2)} Y^{\prime}(s)\right) \mathrm{d} s=\int_{0}^{\lambda} V_{x}^{(2)} \mathrm{d} x
$$

where the integral on the right-hand side is taken at a constant $y$. Taking the limit as this constant approaches infinity we obtain

$$
\frac{1}{2}=\frac{1}{\lambda} \int_{0}^{b}\left(V_{x}^{(2)} X^{\prime}(s)+V_{y}^{(2)} Y^{\prime}(s)\right) \mathrm{d} s .
$$

We map the fluid in region 1 within $-\lambda / 2<x<\lambda / 2$ from the $(z=x+\mathrm{i} y)$-plane into the interior of an annular region of the $\zeta$-plane by the transformation

$$
\zeta=\exp (-2 \mathrm{i} \pi z / \lambda)
$$

We seek the complex velocity $W=u-\mathrm{i} v$ as an analytic function of $\zeta$. Since $W$ is periodic in $x$ with period $\lambda$, it gives rise to a single-valued analytic function of $\zeta$. 
The boundaries of the annular region consist of the free surface and its image in the bottom. We apply Cauchy's integral equation formula to the function $W=u-\mathrm{i} v$ in the annular region of the $\zeta$-plane. This yields

$$
W(\zeta)=-\frac{1}{\pi \mathrm{i}} \oint_{C_{0}} \frac{W(\mu)}{\mu-\zeta} \mathrm{d} \mu
$$

where $\zeta$ is on the free surface and $C_{0}$ denotes the boundaries of the annular region. The integral in (3.13) is a Cauchy-principal value.

Let $\beta=2 \pi / \lambda, V_{1}=u(s) X^{\prime}(s)+v(s) Y^{\prime}(s), V_{2}=u(s) Y^{\prime}(s)-v(s) X^{\prime}(s), Y_{ \pm}=Y(r) \pm Y(s)$, and $X_{ \pm}=X(r) \pm X(s)$. Then taking the real part of (3.13) yields, after some algebra,

$$
\begin{aligned}
\frac{\lambda}{2} u(r)= & \int_{0}^{b / 2} \frac{V_{1}\left(1-\mathrm{e}^{\beta Y_{-}} \cos \left(\beta X_{+}\right)\right)-V_{2}\left(\mathrm{e}^{\beta Y_{-}} \sin \left(\beta X_{+}\right)\right)}{1+\mathrm{e}^{2 \beta Y_{-}}-2 \mathrm{e}^{\beta Y_{-}} \cos \left(\beta X_{+}\right)} \mathrm{ds} \\
& +\int_{0}^{b / 2} \frac{V_{1}\left(1-\mathrm{e}^{\beta Y_{-}} \cos \left(\beta X_{-}\right)\right)+V_{2}\left(\mathrm{e}^{\beta Y_{-}} \sin \left(\beta X_{-}\right)\right)}{1+\mathrm{e}^{2 \beta Y_{-}}-2 \mathrm{e}^{\beta Y_{-}} \cos \left(\beta X_{-}\right)} \mathrm{ds} \\
& -\int_{0}^{b / 2} \frac{V_{1}\left(1-\mathrm{e}^{\beta Y_{+}} \cos \left(\beta X_{-}\right)\right)-V_{2}\left(\mathrm{e}^{\beta Y_{+}} \sin \left(\beta X_{-}\right)\right)}{1+\mathrm{e}^{2 \beta Y_{+}}-2 \mathrm{e}^{\beta Y_{+}} \cos \left(\beta X_{-}\right)} \mathrm{ds} \\
& -\int_{0}^{b / 2} \frac{V_{1}\left(1-\mathrm{e}^{\beta Y_{+}} \cos \left(\beta X_{+}\right)\right)+V_{2}\left(\mathrm{e}^{\beta Y_{+}} \sin \left(\beta X_{+}\right)\right)}{1+\mathrm{e}^{2 \beta Y_{+}}-2 \mathrm{e}^{\beta Y_{+}} \cos \left(\beta X_{+}\right)} \mathrm{ds} .
\end{aligned}
$$

Similarly we use the fact that $V_{x}^{(1)}-\mathrm{i} V_{y}^{(2)}$ is an analytic function of $z$ satisfying $V_{y}^{(1)}=0$ on $y=0$ to derive the integral equation

$$
\begin{aligned}
\frac{\lambda}{2} V_{x}^{(1)}(r)= & \int_{0}^{b / 2} \frac{A_{1}\left(1-\mathrm{e}^{\beta Y_{-}} \cos \left(\beta X_{+}\right)\right)-A_{2}\left(\mathrm{e}^{\beta Y_{-}} \sin \left(\beta X_{+}\right)\right)}{1+\mathrm{e}^{2 \beta Y_{-}}-2 \mathrm{e}^{\beta Y_{-}} \cos \left(\beta X_{+}\right)} \mathrm{ds} \\
& +\int_{0}^{b / 2} \frac{A_{1}\left(1-\mathrm{e}^{\beta Y_{-}} \cos \left(\beta X_{-}\right)\right)+A_{2}\left(\mathrm{e}^{\beta Y_{-}} \sin \left(\beta X_{-}\right)\right)}{1+\mathrm{e}^{2 \beta Y_{-}}-2 \mathrm{e}^{\beta Y_{-}} \cos \left(\beta X_{-}\right)} \mathrm{ds} \\
& -\int_{0}^{b / 2} \frac{A_{1}\left(1-\mathrm{e}^{\beta Y_{+}} \cos \left(\beta X_{-}\right)\right)-A_{2}\left(\mathrm{e}^{\beta Y_{+}} \sin \left(\beta X_{-}\right)\right)}{1+\mathrm{e}^{2 \beta Y_{+}}-2 \mathrm{e}^{\beta Y_{+}} \cos \left(\beta X_{-}\right)} \mathrm{ds} \\
& -\int_{0}^{b / 2} \frac{A_{1}\left(1-\mathrm{e}^{\beta Y_{+}} \cos \left(\beta X_{+}\right)\right)+A_{2}\left(\mathrm{e}^{\beta Y_{+}} \sin \left(\beta X_{+}\right)\right)}{1+\mathrm{e}^{2 \beta Y_{+}}-2 \mathrm{e}^{\beta Y_{+}} \cos \left(\beta X_{+}\right)} \mathrm{ds} .
\end{aligned}
$$

Here $A_{1}=V_{x}^{(1)}(s) X^{\prime}(s)+V_{y}^{(1)}(s) Y^{\prime}(s)$ and $A_{2}=V_{x}^{(1)}(s) Y^{\prime}(s)-V_{y}^{(1)}(s) X^{\prime}(s)$

Next we map the fluid in region 2 within a wavelength $-\lambda / 2<x<\lambda / 2$ from the $z$-plane into the interior of the unit circle by the transformation

$$
\zeta=\exp (2 \mathrm{i} \pi z / \lambda) \text {. }
$$

Applying the Cauchy integral formula to the function $V_{x}^{(2)}-\mathrm{i} V_{y}^{(2)}$ in the $\zeta$-plane we obtain

$$
\begin{aligned}
V_{x}^{(2)}(t)= & \frac{2}{\lambda} \operatorname{Im} \int_{0}^{b} \frac{\left[V_{x}^{(2)}-\mathrm{i} V_{y}^{(2)}\right]\left[\mathrm{i} X^{\prime}(s)-Y^{\prime}(s)\right]}{1-\exp (2 \mathrm{i} \pi / \lambda)(X(t)-X(s))-2 \pi / \lambda(Y(t)-Y(s)))} \mathrm{d} s \\
& +\frac{2}{\lambda} \operatorname{Im} \int_{0}^{b} \frac{\left[V_{x}^{(2)}+\mathrm{i} V_{y}^{(2)}\right]\left[\mathrm{i} X^{\prime}(s)+Y^{\prime}(s)\right]}{1-\exp (2 \mathrm{i} \pi / \lambda(X(t)+X(s))-2 \pi / \lambda(Y(t)-Y(s)))} \mathrm{d} s
\end{aligned}
$$

where Im denotes the imaginary part. 
We now use (2.15) and (2.16) to express $V_{x}^{(1)}$ and $V_{y}^{(1)}$ in terms of $V_{x}^{(2)}$ and $V_{y}^{(2)}$ as

$$
\begin{aligned}
& V_{x}^{(1)}=\frac{1}{1+S_{x}^{2}}\left[V_{y}^{(2)}\left(S_{x}-\frac{S_{x}}{\bar{\epsilon}}\right)+V_{x}^{(2)}\left(1+\frac{S_{x}^{2}}{\bar{\epsilon}}\right)\right], \\
& V_{y}^{(1)}=\frac{1}{1+S_{x}^{2}}\left[V_{y}^{(2)}\left(S_{x}^{2}+\frac{1}{\bar{\epsilon}}\right)+V_{x}^{(2)}\left(S_{x}-\frac{S_{x}}{\bar{\epsilon}}\right)\right] .
\end{aligned}
$$

Finally the kinematic boundary condition on the interface gives

$$
u Y^{\prime}(s)-v X^{\prime}(s)=0 .
$$

There are different ways to define the amplitude $h$. One way is to define it as the height between crests and troughs, i.e.

$$
h=Y(0)-Y\left(\frac{1}{2} b\right) \text {. }
$$

Another way is to define it as the $L_{2}$ norm of $Y-\bar{d}$, i.e.

$$
h=\left[\int_{0}^{b}[Y(s)-\bar{d}]^{2} X^{\prime}(s) \mathrm{d} s\right]^{1 / 2} .
$$

The first one is used in the description of the results.

This completes the reformulation of the problem as a system of integro-differential equations. We seek six functions $X^{\prime}(s), Y^{\prime}(s), u, v, V_{x}^{(2)}, V_{y}^{(2)}$ and three parameters $\alpha$, $B$ and $b$ satisfying (3.2), (3.3), (3.4), (3.14), (3.15), (3.17), (3.18), (3.19) (3.20), (3.21) (or (3.22)). These equations are solved numerically in $\S 5$ but before doing this we develop the solution in the limit of long waves.

\section{Asymptotic nonlinear long-wave solutions}

In what follows we analyse the problem when the thickness of the fluid layer is small compared with the length of the wave. In terms of the non-dimensional equations introduced earlier, this limit can be studied by the introduction of a small parameter $\delta$, say, such that the free surface is given by

$$
S(x, t)=\delta \tilde{S}(x, t)
$$

Physically, $\delta$ is the ratio of the dimensional mean layer thickness to the length of the waves. An asymptotic solution is sought in this limit starting from the exact integral formulation and boundary conditions. An alternative way, which utilizes the scaled equations and uses order-of-magnitude arguments along with matched asymptotic expansions to derive the leading-order solution, can be found in Tilley et al. (2001) (see also Savettaseranee et al. 2003 for the viscous problem). We retain the timedependence in order to compare with the analysis of Tilley et al. (2001). Another difference between the present analysis and that of Tilley et al. (2001) is that $L$ is used to non-dimensionalize lengths instead of $d$. The asymptotic scalings are consequently different but the final evolution equations are identical. The former approach is more natural in making comparisons with the numerical solutions of the full problem when $\lambda \gg 1$.

We introduce a stretched normal coordinate in region 1:

$$
\begin{aligned}
& \text { Region 1: } x \text { unchanged, } y=\delta \zeta, \\
& \text { Region 2: } x \text { unchanged, } y \text { unchanged, }
\end{aligned}
$$


with $\zeta$ an order-one variable. The electric potentials for small $\delta$ expand as follows:

$$
\begin{aligned}
& V^{(1)}=\frac{1}{2} x+\delta \tilde{V}^{(1)}(x, \zeta, t)+O\left(\delta^{3}\right), \\
& V^{(2)}=\frac{1}{2} x+\delta \tilde{V}^{(2)}(x, y, t)+O\left(\delta^{3}\right) .
\end{aligned}
$$

Note that the expansions proceed in $\delta^{2}$ due to the form of the scaled equation for the voltage in region 1 which is

$$
V_{x x}^{(1)}+\frac{1}{\delta^{2}} V_{\zeta \zeta}^{(1)}=0
$$

Substitution of (4.4) into (4.6) and use of the symmetry condition $V_{\zeta}^{(1)}(x, 0, t)=0$, gives

$$
\tilde{V}^{(1)}=\tilde{V}_{0}^{(1)}(x, t)
$$

This result implies that $V_{y}^{(1)}=O\left(\delta^{2}\right)$ in region 1, an estimate which is used next.

We proceed by applying the transformations (4.2) and (4.3) and substituting the expansions (4.4)-(4.5) into the voltage boundary conditions (2.15) and (2.16). In doing this we evaluate at $\zeta=\tilde{S}$ for quantities relating to region 1 (note that $\partial / \partial y \rightarrow(1 / \delta)(\partial / \partial \zeta)$ in region 1$)$, and $y=\delta \tilde{S}$ for quantities evaluated using region 2 variables - vertical derivatives remain of order one in region 2 . We obtain

$$
\begin{aligned}
\bar{\epsilon}\left[O\left(\delta^{2}\right)-\delta \tilde{S}_{x}\left(\frac{1}{2}+O(\delta)\right)\right] & =\left[\delta \tilde{V}_{y}^{(2)}-\delta \tilde{S}_{x}\left(\frac{1}{2}+O(\delta)\right)\right]_{y=\delta \tilde{S}}, \\
\frac{1}{2}+\delta \tilde{V}_{0 x}^{(1)}+O\left(\delta^{2}\right) & =\left[\frac{1}{2}+\delta \tilde{V}_{x}^{(2)}+O\left(\delta^{2}\right)\right]_{y=\delta \tilde{S}} .
\end{aligned}
$$

In the limit $\delta \rightarrow 0$ we replace the leading-order terms from the right-hand sides of (4.8) and (4.9) by evaluation at $y=0$. This assumes that $\nabla \tilde{V}^{(2)}(x, 0, t)=O(1)$, which is confirmed a posteriori. The following relations are then obtained:

$$
\begin{aligned}
\left.\tilde{V}_{y}^{(2)}\right|_{y=0} & =\frac{1}{2}(1-\epsilon) \tilde{S}_{x}, \\
\left.\tilde{V}_{x}^{(2)}\right|_{y=0} & =\tilde{V}_{0 x}^{(1)} .
\end{aligned}
$$

The leading-order boundary conditions are needed in the Bernoulli equation (2.14) which is considered next. The fluid potential $\phi$ in region 1 satisfies the scaled Laplace equation

$$
\phi_{x x}+\frac{1}{\delta^{2}} \phi_{\zeta \zeta}=0
$$

The expansion is

$$
\phi=\phi_{0}+\delta^{2} \phi_{1}+\ldots,
$$

and the solutions follow as

$$
\begin{aligned}
\phi_{0} & =\phi_{0}(x, t), \\
\phi_{1 \zeta} & =-\zeta \phi_{0 x x} .
\end{aligned}
$$

In deriving these solutions we have used symmetry at $\zeta=0$. Inspection of the Bernoulli equation (2.14) and the expressions (2.17)-(2.19) for the electric stresses suggests that $\mathscr{M}_{22}$ dominates in the limit $\delta \ll 1$; introducing the scalings (4.2) and (4.3) with the expansions (4.4)-(4.5) gives

$$
\left[\mathscr{M}_{22}\right]_{2}^{1}=\bar{\epsilon}\left[-\frac{1}{4}-\delta \tilde{V}_{0 x}^{(1)}+\ldots\right]_{\zeta=\tilde{S}}-\left[-\frac{1}{4}-\delta \tilde{V}_{x}^{(2)}+\ldots\right]_{y=\delta \tilde{S}} .
$$


Using condition (4.11) above, and selecting the largest terms in (4.16) gives

$$
\left[\mathscr{M}_{22}\right]_{2}^{1}=\delta(1-\bar{\epsilon})\left[\tilde{V}_{x}^{(2)}\right]_{y=0}+\frac{1}{4}(1-\bar{\epsilon})+\ldots
$$

We note that even though the constant term is larger, it does not play a role since the Bernoulli equation is differentiated with respect to $x$ to obtain the desired evolution equation. The evolution equation is found by considering the largest terms in (2.14), noting that we are evaluating at $S=\delta \tilde{S}$ and all fluid potential terms are calculated using region 1 variables. The resulting expression, after a differentiation with respect to $x$, becomes

$$
\phi_{0 x t}+\phi_{0 x} \phi_{0 x x}+\frac{1}{2} \delta(1-\bar{\epsilon})\left[\tilde{V}_{x x}^{(2)}\right]_{y=0}=\delta \tilde{S}_{x x x} .
$$

Equation (4.18) contains a non-local contribution due to the electric field in the potential region above the fluid layer. The remaining task is to calculate this in terms of the interface position.

One way to accomplish this is to note that the function $\tilde{V}_{x}^{(2)}-\mathrm{i} \tilde{V}_{y}^{(2)}$ is an analytic function of the complex variable $z=x+\mathrm{i} y$. We apply Cauchy's theorem also to the analytic function $\tilde{V}_{x x}^{(2)}-\mathrm{i} \tilde{V}_{y x}^{(2)}$ on a contour bounding region 2 (it is easier to consider $-\infty<x<\infty$ in this and apply periodicity at the end). The contour is rectangular with its upper part located at $y=Y_{0}$, and as $Y_{0} \rightarrow \infty$ the contribution to the integral tends to zero since $\tilde{V}_{x x}^{(2)}-\mathrm{i} \tilde{V}_{y x}^{(2)} \rightarrow 0$ there. In addition, the contributions from the two vertical parts of the contour cancel and evaluating as $y \rightarrow 0$ gives

$$
\tilde{V}_{x x}^{(2)}(x, 0)-\mathrm{i} \tilde{V}_{y x}^{(2)}(x, 0)=\frac{1}{\mathrm{i} \pi} \mathrm{PV} \int_{-\infty}^{\infty} \frac{\tilde{V}_{x x}^{(2)}\left(x^{\prime}, 0\right)-\mathrm{i} \tilde{V}_{y x}^{(2)}\left(x^{\prime}, 0\right)}{x^{\prime}-x} \mathrm{~d} x^{\prime} .
$$

Take the real part of this expression and differentiate (4.10) with respect to $x$ to eliminate $\tilde{V}_{x y}^{(2)}(x, 0)$; this gives

$$
\left[\tilde{V}_{x x}^{(2)}\right]_{y=0}=\frac{1}{2}(1-\bar{\epsilon})\left[\frac{1}{\pi} \mathrm{PV} \int_{-\infty}^{\infty} \frac{\tilde{S}_{x^{\prime} x^{\prime}}}{x-x^{\prime}} \mathrm{d} x^{\prime}\right] \equiv \frac{1}{2}(1-\bar{\epsilon}) \mathscr{H}\left(\tilde{S}_{x x}\right),
$$

where $\mathscr{H}(\cdot)$ is the Hilbert transform operator. We note that for periodic functions the Hilbert transform becomes

$$
\mathscr{H}\left(\tilde{S}_{x x}\right)=-\mathrm{PV} \int_{-1 / 2}^{1 / 2} \cot \left(\pi\left(x-x^{\prime}\right)\right) \tilde{S}_{x^{\prime} x^{\prime}} \mathrm{d} x^{\prime} .
$$

This is the appropriate form in the present study. The leading-order Bernoulli equation becomes, then,

$$
\phi_{0 x t}+\phi_{0 x} \phi_{0 x x}-\frac{1}{4} \delta E_{b}(1-\bar{\epsilon})^{2} \mathscr{H}\left(\tilde{S}_{x x}\right)=\delta \tilde{S}_{x x x} .
$$

A second evolution equation follows from the kinematic condition (2.13). Using the scalings and leading-order solutions found here, this becomes

$$
\tilde{S}_{t}+\phi_{0 x} \tilde{S}_{x}+\phi_{0 x x} \tilde{S}=0 .
$$

Re-writing equations (4.22) and (4.23) in terms of the unscaled variables (see (4.1)), we obtain

$$
\begin{gathered}
\phi_{0 x t}+\phi_{0 x} \phi_{0 x x}-\frac{1}{4} E_{b}(1-\bar{\epsilon})^{2} \mathscr{H}\left(S_{x x}\right)=S_{x x x}, \\
S_{t}+\phi_{0 x} S_{x}+\phi_{0 x x} S=0 .
\end{gathered}
$$

It remains to cast the equations into the computational format of our arbitrary amplitude calculations. This is achieved by looking for steady states and introducing 
a uniform stream of speed $c$. Writing

$$
\phi_{0}=c x+\phi, \quad \frac{\partial}{\partial t} \equiv 0,
$$

gives the system

$$
\begin{aligned}
c \phi_{x x}+\phi_{x} \phi_{x x}-\frac{1}{4}(1-\bar{\epsilon})^{2} E_{b} \mathscr{H}\left(S_{x x}\right) & =S_{x x x}, \\
c S_{x}+S_{x} \phi_{x}+S \phi_{x x} & =0 .
\end{aligned}
$$

Equations (4.27)-(4.28) are solved numerically in $\S 5$ and results are compared with the full simulations as the wavelength $\lambda$ is increased.

We close this section by giving the wave speed $c$ for the linear theory of the system (4.27) and (4.28). Linearizing about the exact solution $(\phi, S)=(0, \bar{d})$ and using the properties of the Hilbert transform, it is easy to calculate that linear sine waves of wavenumber $k$ travel with speeds

$$
c^{2}=\bar{d} k^{2}+\frac{1}{4} \bar{d} E_{b}(1-\bar{\epsilon})^{2}|k| .
$$

This dispersion relation is used to check the numerical work that follows.

\section{Numerical method}

\subsection{Fully nonlinear problem}

In this section we outline the numerical method used to solve the system of integrodifferential equations derived in $\S 3$.

We define equally spaced mesh points over the interval $\left[0, \frac{1}{2} b\right]$ by the formulae

$$
\begin{gathered}
s_{I}=\frac{b}{2(N-1)}(I-1), \quad I=1, \ldots, N, \\
s_{I+1 / 2}=\frac{\left(s_{I+1}+s_{I}\right)}{2}, \quad I=1, \ldots, N-1 .
\end{gathered}
$$

We also introduce the $6 N$ unknowns $X_{I}^{\prime}=X^{\prime}\left(s_{I}\right), Y_{I}^{\prime}=Y^{\prime}\left(s_{I}\right), u_{I}=u\left(s_{I}\right), v_{I}=v\left(s_{I}\right)$, $V_{x}^{I}=V_{x}^{(2)}\left(s_{I}\right)$ and $V_{y}^{I}=V_{y}^{(2)}\left(s_{I}\right)$.

We satisfy (3.1), (3.4) and (3.20) at the mesh points (5.1). This leads to $3 N$ equations. $2 N-4$ equations are obtained by satisfying (3.14) at the mesh points (5.2), $I=1, \ldots, N-2$ and (3.15) at the mesh points (5.2), I=2,.,$N-1$. The integrals in (3.14) and (3.15) are approximated by the trapezoidal rule with a summation over the mesh points (5.1). The symmetry of the quadrature and of the discretization enables us to evaluate the Cauchy principal values as if they were ordinary integrals. Similarly we satisfy (3.15) at the mesh points (5.1), $I=1, \ldots, N-2$ with a summation over the mesh points (5.2). Seven more equations are obtained by setting $v_{1}=v_{N}=V_{y}^{1}=V_{y}^{N}=0, X(n)=0.5$ and by imposing (3.11) and (3.7). One more equation is given by (3.21) or (3.22) with $h$ prescribed. The last equation expresses $V_{y}^{N}$ in terms of $V_{y}^{I}, I=N-4, \ldots, N-1$ by a four-point extrapolation formula. This gives a system of $6 N+3$ nonlinear algebraic equations for the $6 N+3$ unknowns $B, c$, $\alpha$ and $X_{I}^{\prime}, Y_{I}^{\prime}, u_{I}, v_{I}, V_{x}^{I}, V_{y}^{I}, I=1, \ldots, N$. This system is solved by Newton's method.

\subsection{Long-wave approximation}

We now solve the system (4.27)-(4.28) by a numerical method similar to that of $\S 5.1$. We introduce the mesh points

$$
X_{I}=\frac{\lambda(I-1)}{2(N-1)}, \quad I=1, \ldots, N,
$$




$$
X_{I+1 / 2}=\frac{X_{I+1}+X_{I}}{2}, \quad I=1, \ldots, N-1,
$$

and the unknowns

$$
\phi_{I}^{\prime}=\phi_{x}\left(X_{I}\right), \quad S_{I}^{\prime}=S_{x}\left(X_{I}\right), \quad I=1, \ldots, N .
$$

Next we evaluate $\phi_{x}, \phi_{x x}, S_{x x x}, S_{x}$ at the mesh points (5.4) and $S_{x x}$ at the mesh points (5.3) by four-point difference formulae. We evaluate $S\left(X_{I+1 / 2}\right)$ by integrating $S_{I}^{\prime}$ numerically with the condition $S(0)=\alpha$.

We obtain $2 N-3$ equations by satisfying (4.27) at the mesh points $(5.4), I=1, \ldots$, $N-1$ and (4.28) at the mesh points (5.4), $I=1, \ldots, N-2$. As in $\S 5.1$ the Cauchy principal value in (4.27) is evaluated by the trapezoidal rule with a summmation over the mesh points (5.3). Two more equations are given by $S_{1}^{\prime}=S_{N}^{\prime}=0$. The last three equations are obtained by imposing

$$
\begin{gathered}
\frac{1}{\lambda} \int_{0}^{\lambda / 2} S \mathrm{~d} x=\frac{\bar{d}}{2}, \\
\frac{1}{\lambda} \int_{0}^{\lambda / 2} \phi_{x} \mathrm{~d} x=\frac{\lambda}{2},
\end{gathered}
$$

and by fixing the amplitude $h$ by the equation

$$
S\left(X_{1}\right)-S\left(X_{N}\right)=h .
$$

This yields a system of $2 N+2$ equations for the $2 N+2$ unknowns (5.5), $\alpha$ and $c_{0}$. This system is solved by Newton's method.

\section{Discussion of the results}

We used the numerical schemes described in $\S 5$ to compute solutions for various values of $\lambda, h, \bar{\epsilon}$ and $E_{b}$. We also assume, without loss of generality, that $\bar{d}=1$. Most of the results presented were obtained with $N=100$. We repeated the calculations with larger and smaller values of $N$ and checked that all the results presented are independent of $N$ within graphical accuracy.

When $\bar{\epsilon}=1,(2.15)$ and (2.16) imply $V_{x}^{(1)}=V_{x}^{(2)}$ and $V_{y}^{(1)}=V_{y}^{(2)}$. It then follows that the last term on the left-hand side of (2.14) vanishes and the problem reduces to the capillary waves (without electric fields) considered by Crapper (1957) and Kinnersley (1976). A typical profile in this case and for $h=0.5$, is shown in figure 2 (solid curve). Since the wavelength is relatively small compared to the thickness of the layer $(\lambda=2 \pi / 5)$, this solution is close to the infinite-depth results of Crapper (1957). The profile is clearly nonlinear with a sharp trough and a rounded crest. As the amplitude is further increased the profile approaches a limiting configuration with a trapped bubble at its trough (not shown here). The other profiles in figure 2 show the influence of the relative permittivity of the fluid on waves produced for a fixed applied electric field. Here $E_{b}=3$ and $h=0.5$. As $\bar{\epsilon}$ decreases below 1 , that is the permittivity of the fluid layer is smaller than that of the surrounding medium, the profiles become steeper. As $\bar{\epsilon}$ increases above 1, the profiles become closer to linear sine waves and the electric field suppresses the wave steepness seen in its absence.

In figure 3 , we present computed values of $c$ versus $E_{b}$ for $\lambda=4 \pi, 12 \pi$ and $20 \pi$. The value of the relative permittivity is set to $\bar{\epsilon}=1.5$ and the amplitude of the travelling waves is taken to be $h=0.1$. Figure 3 consists of a pair of lines for each of the 


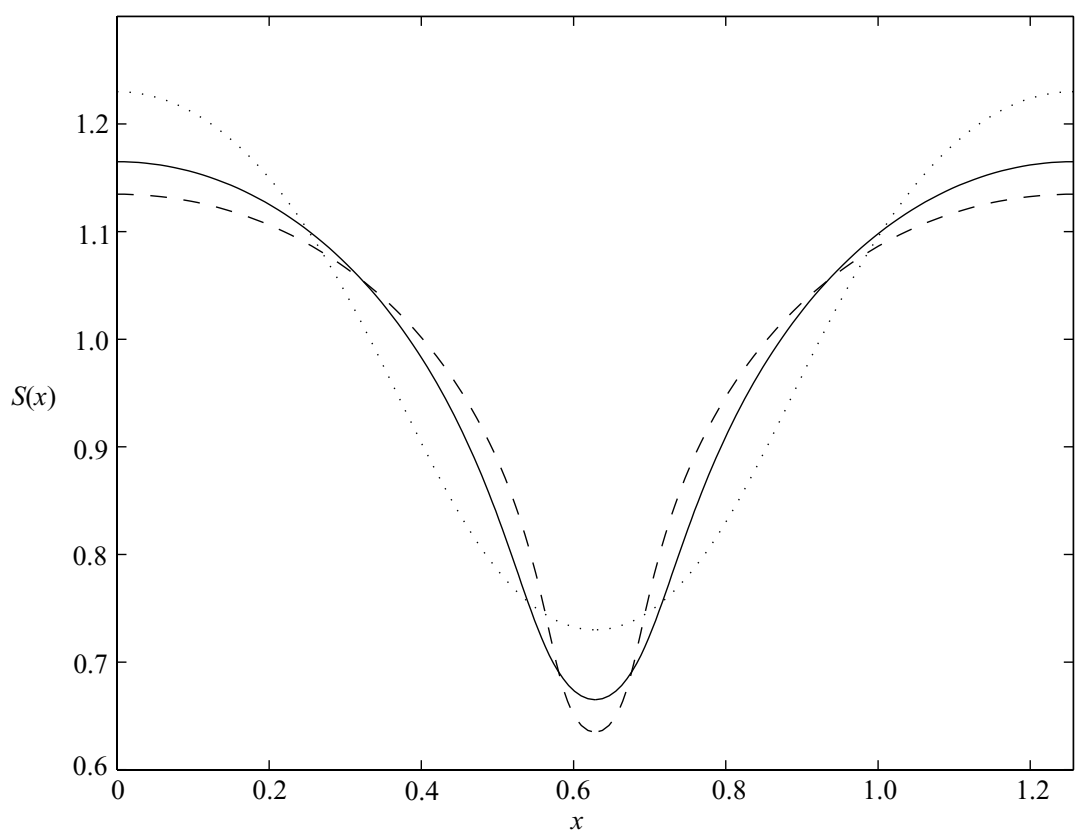

FIGURE 2. Shapes of nonlinear travelling waves for different relative permittivities. The electric capillary number is $E_{b}=3$, the wave amplitude is $h=0.5, \lambda=2 \pi / 5$ and the dimensionless thickness is 2 (i.e. $\bar{d}=1$ ). Solid line, $\bar{\epsilon}=1$; dashed line, $\bar{\epsilon}=0.075$; dotted line, $\bar{\epsilon}=4$.

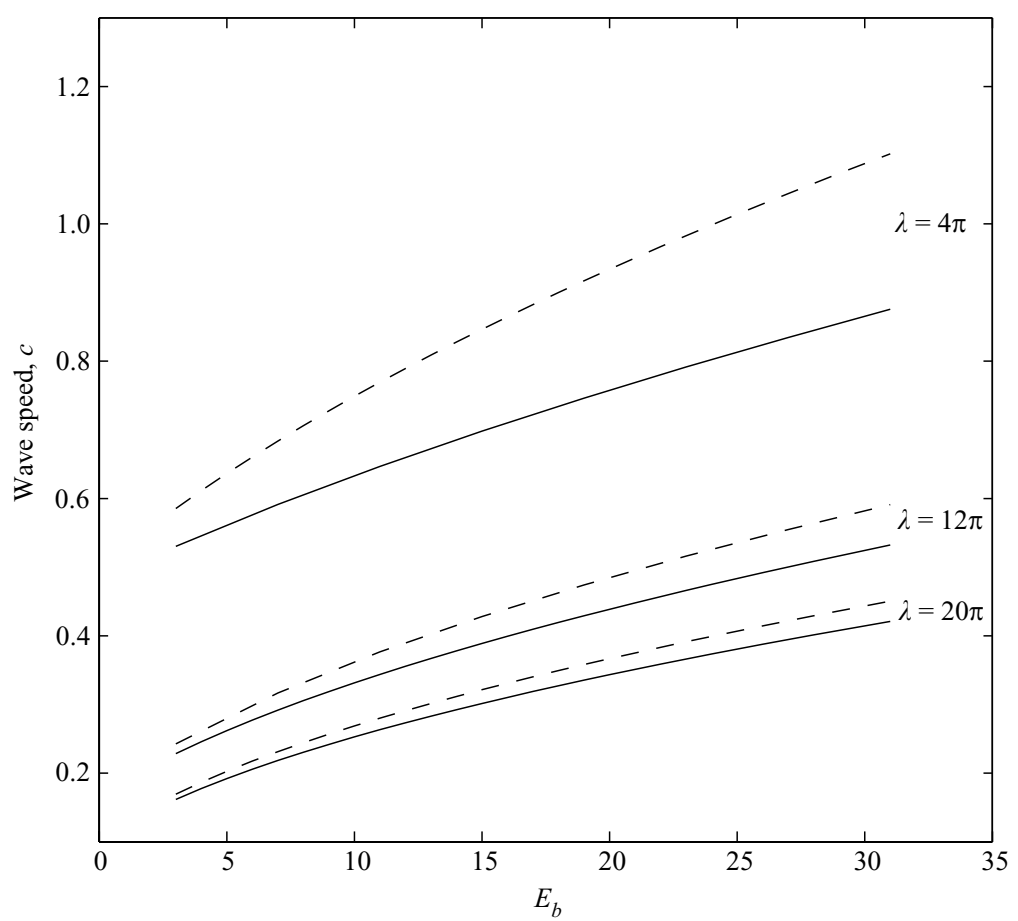

FIGURE 3. Travelling wave speed $c$ versus the electric capillary number $E_{b} . h=0.1, \bar{\epsilon}=1.5$, $\bar{d}=1$. Dashed curves are weakly nonlinear results and solid curves are fully nonlinear. The wavelength $\lambda$ is given for each set of results. 


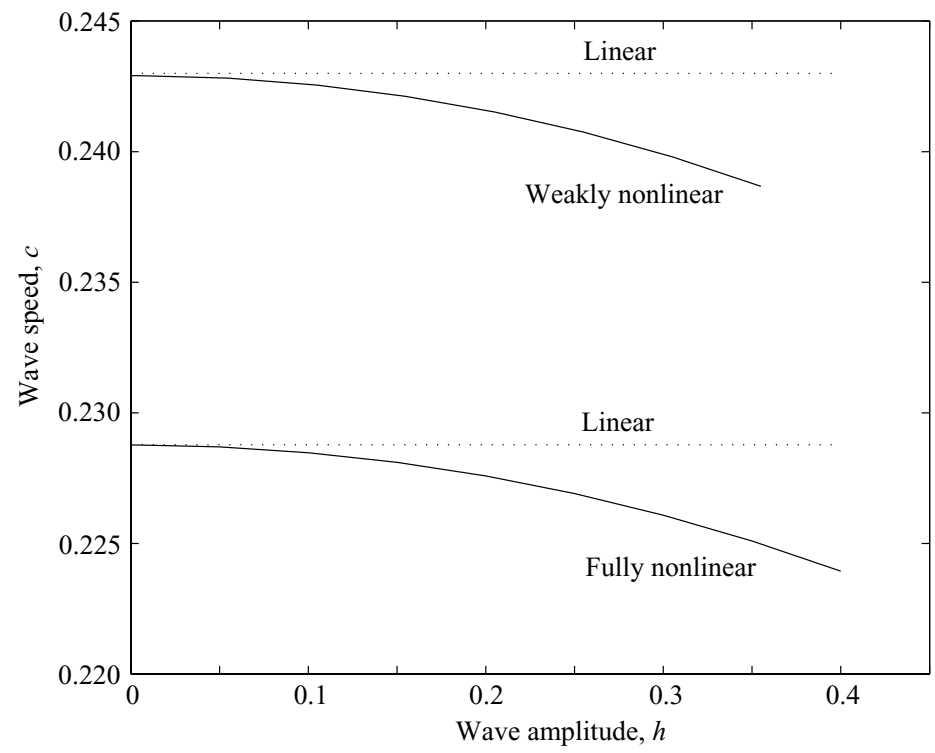

FIGURE 4. Travelling wave speed $c$ versus wave amplitude $h . E_{b}=3, \lambda=12 \pi, \bar{\epsilon}=1.5, \bar{d}=1$. Top curves, long-wave model. Lower curves, fully nonlinear problem.

wavelengths $4 \pi, 12 \pi$ and $20 \pi$, respectively. In each group, the solid line represents the fully nonlinear results obtained by using the scheme of $\S 5.1$ whereas the broken line shows the results according to the asymptotic model of $\S 4$ and calculated using the scheme of $\S 5.2$. It can be seen that agreement between the fully nonlinear calculations and those based on the long-wave model becomes increasingly better as $\lambda$ increases (large $\lambda$ corresponds to small $\delta$ which is necessary for the validity of the asymptotic equations). The ratio of the absolute errors of the asymptotic and fully nonlinear speeds for the cases $\lambda=20 \pi$ and $12 \pi$ is 0.5 within an error of $6 \%$ or less for the whole range of values of $E_{b}$ represented in figure 3 . These results indicate that the long-wave model is very good for wavelengths larger than about $10 \pi$.

In figure 4, we present values of the wave speed $c$ versus the wave amplitude $h$ for a fixed electric capillary number $E_{b}=3$ and wavelength $\lambda=12 \pi$. The value of the relative permittivity is $\bar{\epsilon}=1.5$. Results are shown for both the weakly nonlinear problem based on the equations derived in $\S 4$, and calculations using the fully nonlinear equations and the computational methods described here. The upper solid curve corresponds to the weakly nonlinear solution, whereas the lower solid curve is the fully nonlinear solution. The dotted curves represent the wave speed which is obtained in the linear regime with the wavenumber fixed to the value $k=2 \pi / \lambda=\frac{1}{6}$, chosen for the computations. The linear speed for the long-wave model has been given in equation (4.29) and the straight line follows by substituting $k=\frac{1}{6}, E_{b}=3$ and $\bar{\epsilon}=1.5$ in this expression (the result is a straight line because linear theory is independent of the amplitude). The analogous dotted curve that depicts the linear speed of the full problem is calculated using the dispersion relation (2.24) with $\bar{d}=1$, and substituting the appropriate values as above, noting that the wave speed $c=\omega / k$. We note that the two curves are different and this is to be expected because the wavenumber $k=\frac{1}{6}$ used in the numerical experiments is finite. As $k$ is reduced, the linear theories of the full problem and the weakly nonlinear problem asymptote to the same value of $c$ which is given in equation (4.29). Owing to the algebraic decay 
rates to this limit we have not followed this further. Note also that these results serve as an additional check on the accuracy of our numerical work.

\section{Conclusions}

In this article we have studied nonlinear interfacial waves of arbitrary amplitude and wavelength on electrified liquid layers. The electric field acts in a direction parallel to the undisturbed layer and we have included the effects of surface tension. Travelling waves have been constructed using boundary integral techniques and the results compared with those of an asymptotic long-wave theory. The long-wave theory is carried out using the integral equation formulation of the problem and results in two coupled evolution equations. These were also derived by Tilley et al. (2001) using different matched asymptotics methods and a different rescaling; the present rescaling is more natural in the sense that the long-wave results can be compared directly with the full numerical calculations. Such comparisons are made extensively here, with the finding that agreement between the two is excellent if the wavelength of the full calculations is sufficiently long. For example, when the relative permittivity $\bar{\epsilon}=1.5$ and the wave amplitude is 0.1 , the travelling wave speeds of the full calculations agree very well with those of the long wave ones for all $E_{b}$ (a measure of the imposed field) studied in a range between 3 and 30, when the wavelength is larger than about $10 \pi$ (this corresponds to an aspect ratio of about 0.03 ).

Our results show that the electric field can have a pronounced effect on the shapes and speeds of interfacial waves. We have established numerically that, all else being the same, when the relative permittivity $\bar{\epsilon}$ becomes larger than 1 (that is the permittivity in fluid region 1 is larger than that of the surrounding medium), the nonlinear waves lose their steepness and resemble sine waves. At the same time the wave speed increases. On the other hand, when $\bar{\epsilon}<1$ (region 2 has larger permittivity than region 1), the waves steepen relative to the zero electric field case, and the wave speed decreases. These results are summarized in figures 2 and 3 - note that $E_{b}$ and $\bar{\epsilon}$ appear independently in the equations even though the Maxwell stress contribution proportional to $E_{b}(1-\bar{\epsilon})^{2}$ appears to be the controlling parameter. Figure 3 also shows that the electric field can be used to control the speed of the nonlinear waves since the latter has been established from our numerical work to be a monotonically increasing function of the former.

Considering real physical systems, we see that the scenario predicted for $\bar{\epsilon}>1$ is probably the most appropriate for our problem. Values of dielectric constants have been given in the experimental study of Burcham \& Saville (2000) who study liquid bridge formation when an axial field acts in the absence of gravity. The surrounding phase is a gas (sulphur hexafluoride) while the bridge fluid is a certain oil (see table 1 of Burcham \& Saville 2000). In all those systems, the value of $\bar{\epsilon}$ ranges from 2.74 for the highest viscosity fluid (a silicon oil $12 \mathrm{M}$ ) to 5.24 for the lowest viscosity fluid (castor oil/eugenol). Two-phase liquid-liquid systems can be used that have values of $\bar{\epsilon}<1$ (for example silicon oil $1 \mathrm{M}$ surrounded by a less viscous castor oil/eugenol see table 1 of Burcham \& Saville (2000)) which gives $\bar{\epsilon}=0.53$. Our theory needs to be modified in this case, however, to take into account the fluid dynamics of the surrounding phase. This is the subject of future research.

The work undertaken here is two-dimensional and restricted to waves which are symmetric with respect to the centreline. In the absence of electric fields, the work of Kinnersley (1976) shows that there are in addition antisymmetric waves (see also Taylor 1959 for a linear theory). The linear theory for antisymmetric waves 
follows that of $\S 2.2$ and leads to (2.24) with the tanh replaced by coth. We are now investigating the possible existence of antisymmetric waves in the presence of electric fields, see Papageorgiou \& Vanden-Broeck (2004).

It is also of interest to consider three-dimensional extensions and in particular axisymmetric systems which can be found in experiments. Axisymmetric capillary waves were considered by Vanden-Broeck, Miloh \& Spivack (1998) and inclusion of axial electric fields in those flows is the subject of ongoing work.

The work of D.T.P. and J.-M. V.-B. was supported by the National Science Foundation Grant numbers DMS-0072228 and DMS-0204808, respectively. The work was also supported by EPSRC and the Leverhulme Trust.

\section{REFERENCES}

Al-Sibai, F., Leefken, A. \& Renz, U. 2002 Local and instantaneous distribution of heat transfer rates through wavy films. Intl J. Therm. Sci. 41, 658-663.

Burcham, C. L. \& Saville, D. A. 2000 The electrohydrodynamic stability of a liquid bridge: Microgravity experiments on a bridge suspended in a dielectric gas. J. Fluid Mech. 405, 37-56.

Chen, B. \& Saffman, P. G. 1979 Steady gravity-capillary waves on deep water. Stud. Appl. Maths 60, 183-210.

Crapper, G. D. 1957 An exact solution for progressive capillary waves of arbitrary amplitude. J. Fluid Mech. 2, 532-540.

Crowdy, D. G. 1999 Exact solutions for steady capillary waves on a fluid annulus. J. Nonlinear Sci. 9, 615-640.

EL-SAYED, M. F. 1999 Electro-aerodynamic instability of a thin dielectric liquid sheet sprayed with an air stream. Phys. Rev. E 60, 7588-7591.

Erneux, T. \& Davis, S. H. 1993 Nonlinear rupture of free films. Phys. Fluids A 5, 1117-1122.

GonZalez, A. \& CASTEllanos, A. 1996 Nonlinear electrohydrodynamic waves on films falling down an inclined plane. Phys. Rev. E 53, 3573-3578.

Hogan, S. J. 1980 Some effects of surface tension on steep water waves. Part 2. J. Fluid Mech. 96, 417-445.

Hunter, J. K. \& VANDEN-BroecK, J.-M. 1983 Solitary and periodic gravity-capillary waves of finite amplitude. J. Fluid Mech. 134, 205-219.

IDA, M. P. \& Miksis, M. J. 1996 Thin film rupture. Appl. Math. Lett. 9, 35-40.

IDA, M. P. \& Miksis, M. J. $1998 a$ The dynamics of thin films I: General theory. SIAM J. Appl. Maths 58, 456-473.

IdA, M. P. \& Miksis, M. J. $1998 b$ The dynamics of thin films II: Applications. SIAM J. Appl. Maths 58, 474-500.

JACKSON, J. D. 1963 Classical Electrodynamics. John Wiley and Sons.

KANG, Y. \& VANDEN-BRoECK, J.-M. 2000 Gravity-capillary waves in the presence of constant vorticity. Eur. J. Mech. B-Fluids 19, 253-268.

KinNERSLEY, W. 1976 Exact large amplitude capillary waves on sheets of fluid. J. Fluid Mech. 77, 229-241.

Longuet-Higgins, M. S. 1975 Integral properties of periodic gravity waves of finite amplitude. Proc. R. Soc. Lond. A 342, 157-174.

MatsuUCHI, K. 1974 Modulational instability of nonlinear capillary waves on thin liquid films. J. Phys. Soc. Japan 37, 1680-1687.

MatsuuchI, K. 1976 Instability of thin liquid sheet and its break-up. J. Phys. Soc. Japan 41, $1410-1416$.

Mehring, C. \& Sirignano, W. A. 1999 Nonlinear capillary wave distortion and disintegration of thin planar sheets. J. Fluid Mech. 388, 69-113.

Melcher, J. R. \& SchWARZ, W. J. JR 1968 Interfacial relaxation overstability in a tangential electric field. Phys. Fluids 11, 2604-2616.

MiYara, A. 1999 Numerical analysis on flow dynamics and heat transfer of falling liquid films with interfacial waves. Heat and Mass Transfer 35, 298-306. 
Papageorgiou, D. T. \& Vanden-Broeck, J.-M. 2004 Antisymmetric capillary waves in electrified fluid sheets. Eur. J. Appl. Mech. (submitted).

Pugh, M. C. \& Shelley, M. J. 1998 Singularity formation in thin jets with surface tension. Commun. Pure Appl. Maths 51, 733-795.

Savettaseranee, K., Papageorgiou, D. T., Petropoulos, P. G. \& Tilley, B. S. 2003 The effect of electric fields on the rupture of thin viscous films by van der Waals forces. Phys. Fluids 15, $641-652$.

Schwartz, L. W. 1974 Computer extension and analytic continuation of Stokes' expansion for gravity waves. J. Fluid Mech. 62, 553-578.

Schwartz, L. W. \& VANDEN-Broeck, J.-M. 1979 Numerical solution of the exact equations for capillary-gravity waves. J. Fluid Mech. 95, 119-139.

Shmerler, J. \& Mudawar, I. 1987 Effects of interfacial waves on heat transfer to free-falling turbulent liquid films. In Particulate Phenomena and Multiphase Transport, vol. 2 (ed. T. N. Veziroglu), pp. 361-376. Hemisphere.

Stokes, G. G. 1847 On the theory of oscillatory waves. Camb. Trans. 8, 441-473.

TAYLOR, G. I. 1959 The dynamics of thin sheets of fluid. II. Waves on fluid sheets. Proc. R. Soc. Lond. A 253, 296-312.

Tilley, B. S., Petropoulos, P. G. \& Papageorgiou, D. T. 2001 Dynamics and rupture of planar electrified liquid sheets. Phys. Fluids 13, 3547-3563.

Vanden-Broeck, J.-M., Miloh, T. \& Spivack, B. 1998 Axisymmetric capillary waves. Wave Motion 27, 245-256.

VAYnblat, D., Lister, J. R. \& Witelski, T. P. 2001 Rupture of thin viscous films by van der Waals forces: Evolution and self-similarity. Phys. Fluids 13, 1130-1140. 